\title{
Colloidal matter: Packing, geometry, and entropy
}

\section{Citation}

Manoharan, V. N. 2015. Colloidal Matter: Packing, Geometry, and Entropy. Science 349, no. 6251: 1253751-1253751. doi:10.1126/science.1253751.

\section{Published Version}

10.1126/science.1253751

\section{Permanent link}

http://nrs.harvard.edu/urn-3:HUL.InstRepos:30410808

\section{Terms of Use}

This article was downloaded from Harvard University's DASH repository, and is made available under the terms and conditions applicable to Open Access Policy Articles, as set forth at http:// nrs.harvard.edu/urn-3:HUL.InstRepos:dash.current.terms-of-use\#OAP

\section{Share Your Story}

The Harvard community has made this article openly available.

Please share how this access benefits you. Submit a story.

\section{Accessibility}




\title{
Colloidal matter: packing, geometry, and entropy
}

\author{
Vinothan N. Manoharan
}

Harvard John A. Paulson School of Engineering and Applied Sciences and Department of Physics, Harvard University, Cambridge MA 02138 USA

This is the author's version of the work. It is posted here by permission of the AAAS for personal use, not for redistribution. The definitive version was published in $\underline{\text { Science volume } 349 \text { on August 8, 2015. doi: 10.1126/science. } 1253751}$

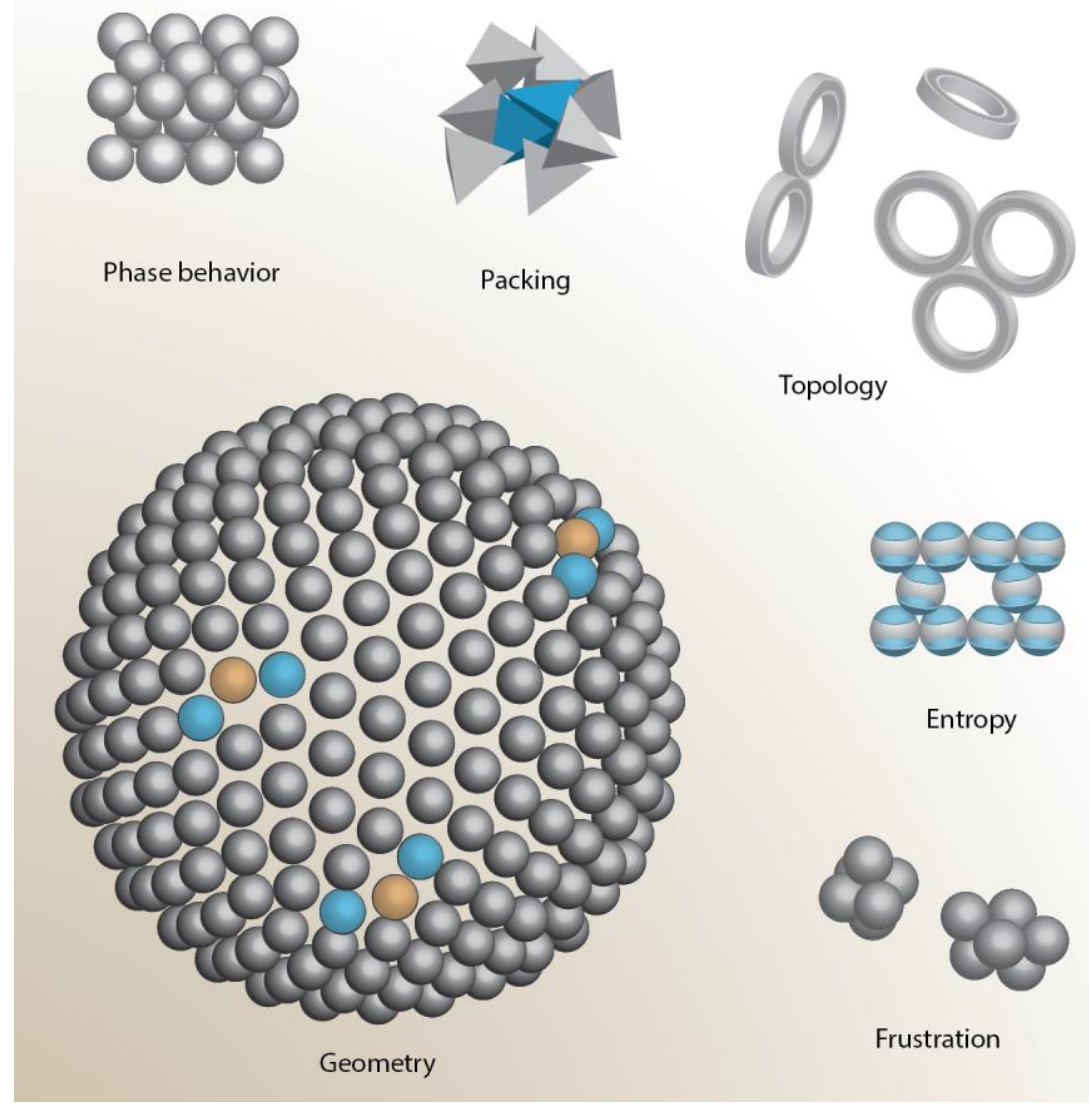

The many dimensions of colloidal matter. The self-assembly of colloids can be controlled by changing the shape, topology, or patchiness of the particles, by introducing attractions between particles, or by constraining them to a curved surface. All of the assembly phenomena illustrated here can be understood from the interplay between entropy and geometrical constraints. 


\section{Summary of this review article}

\section{Background:}

Colloids consist of solid or liquid particles, each about a few hundred nanometers in size, dispersed in a fluid and kept suspended by thermal fluctuations. While natural colloids are the stuff of paint, milk, and glue, synthetic colloids with well-controlled size distributions and interactions are a model system for understanding phase transitions. These colloids can form crystals and other phases of matter seen in atomic and molecular systems, but because the particles are large enough to be seen under an optical microscope, the microscopic mechanisms of phase transitions can be directly observed. Furthermore, their ability to spontaneously form phases that are ordered on the scale of visible wavelengths makes colloids useful building blocks for optical materials such as photonic crystals. Because the interactions between particles can be altered and the effects on structure directly observed, experiments on colloids offer a controlled approach toward understanding and harnessing self-assembly, a fundamental topic in materials science, condensed matter physics, and biophysics.

\section{Advances:}

In the past decade, our understanding of colloidal self-assembly has been transformed by experiments and simulations that subject colloids to geometrical or topological constraints, such as curved surfaces, fields, or the shapes of the particles themselves. In particular, advances in the synthesis of nonspherical particles with controlled shape and directional interactions have led to the discovery of structural transitions that do not occur in atoms or molecules. As a result, colloids are no longer seen as a proxy for atomic systems but as a form of matter in their own right. The wide range of self-assembled structures seen in colloidal matter can be understood in terms of the interplay between packing constraints, interactions, and the freedom of the particles to move - in other words, their entropy. Ongoing research attempts to use geometry and entropy to explain not only structure but dynamics as well. Central to this goal is the question of how entropy favors certain local packings. The incompatibility of these locally-favored structures with the globally-favored packing can be linked to the assembly of disordered, arrested structures such as gels and glasses.

\section{Outlook}

We are just beginning to explore the collective effects that are possible in colloidal matter. The experimentalist can now control interactions, shapes, and confinement, and this vast parameter space is still expanding. Active colloidal systems, dispersions of particles driven by intrinsic or extrinsic energy sources rather than thermal fluctuations, can show nonequilibrium selforganization with complexity rivaling that of biological systems. We can also expect new structural transitions to emerge in "polygamous" DNA-functionalized colloids, which have no equivalent at the molecular scale. New frameworks are needed to predict how all of these variables - confinement, activity, and specific interactions - interact with packing constraints to govern both structure and dynamics. Such frameworks would not only reveal general principles of self-assembly but would also allow us to design colloidal particles that pack in prescribed ways, both locally and globally, thereby enabling the robust self-assembly of optical materials. 


\title{
Colloidal matter: packing, geometry, and entropy
}

\author{
Vinothan N. Manoharan \\ Harvard John A. Paulson School of Engineering and Applied Sciences and Department of \\ Physics, Harvard University, Cambridge MA 02138 USA
}

\begin{abstract}
Colloidal particles with well-controlled shapes and interactions are an ideal experimental system for exploring how matter organizes itself. Like atoms and molecules, these particles form bulk phases such as liquids and crystals. But they are more than just crude analogues of atoms; they are a form of matter in their own right, with complex and interesting collective behavior not seen at the atomic scale. Their behavior is affected by geometrical or topological constraints, such as curved surfaces or the shapes of the particles. Because the interactions between the particles are often short-ranged, we can understand the effects of these constraints using geometrical concepts such as packing. The geometrical viewpoint gives us a window into how entropy affects not only the structure of matter, but also the dynamics of how it forms.
\end{abstract}

\section{Introduction}

A colloid is a dispersion of microscopic particles that are individually unremarkable but collectively interesting. More traditionally, one might say that a colloid is a fluid dispersion, like fog, milk, paint, or glue, where the particles are larger than a molecule but small enough to stay dispersed for days or longer. But defining colloids in the traditional way is a bit like defining puppies as young dogs; though technically accurate, it doesn't quite capture their appeal. The feature of colloids that drives much current research is their collective behavior - their ability to form complex structures and show unusual dynamical transitions. By exploring how these collective effects emerge, we gain insights into general questions of how matter organizes itself, questions that are fundamental to condensed-matter physics, materials science, and even our understanding of life itself.

Many of the things that used to be called colloidal particles are now more often called nanoparticles, the "nano-" prefix highlighting a size scale where unusual electronic, magnetic, or optical properties reside. Meanwhile, the term "colloid" (1) has come to refer to a dispersion of larger particles, perhaps hundreds of nanometers or so, with uniform shapes and a controlled size distribution. Compared to a nanoparticle, an individual colloidal particle (in this narrower definition of the term) has no remarkable material properties. From the perspective of quantum mechanics, it is a blob of macroscopic matter, no more interesting than a baseball.

It is precisely because there is nothing exciting about an individual particle that the collective behavior of colloids is worth studying. Each particle is classical, and its interaction with other particles is generally related to its shape. Yet each is big enough so that we can resolve it from its neighbors under an optical microscope. So when we see these well-characterized, mundane classical particles doing something collectively unexpected-like forming a crystal — it tells us something about the fundamental physics governing that same phenomenon in more complex systems. Indeed, colloids do form crystals, as well as other phases of matter found in atomic and molecular systems, and because the structural length scales of these phases are on the order of 
visible light, they serve as useful model systems for observing the microscopic mechanisms of phase transitions.

But colloids are more than just crude models for atomic systems; they are themselves a form of matter, with interesting collective behavior not seen at the atomic scale. By observing how that behavior is modified by geometrical or topological constraints, such as surfaces, fields, or the shapes of the particles themselves, we garner clues about how order arises in condensed phases, and how it might be disrupted. An enduring mystery of biological systems is how they harness stochastic motion at the molecular level to build ordered structures. Experiments on colloids constitute perhaps the most carefully-controlled approach toward understanding the statistical physics underlying this process, which is called self-assembly. The self-assembly of colloidal particles is well-worth studying on its own merits, since it can be used to make photonic crystals and macroporous materials $(2,3)$, although that is not the focus of this review.

The discussion will focus on the general principles concerning the structure and dynamics of colloidal matter, and how this behavior can be understood in terms of geometry and topology. Two themes emerge. The first is that entropy, an abstract thermodynamic quantity colloquially associated with "disorder," makes its presence felt in unexpected ways, including driving the formation of ordered phases. The second is the relation between packing constraints, which are imposed by the shapes of the particles, and structure and structural transitions. These two themes intersect at one of the frontiers of the field: understanding how entropy and geometry govern not only the structure of matter but also the dynamics of how it forms.

\section{From atoms to model atoms}

To understand why entropy is so important in colloidal dispersions, we first consider a single colloidal particle in a liquid. As Robert Brown showed in 1827 (4), it moves about randomly and incessantly. This Brownian motion, as we now call it, was quantified by Jean Perrin (5), who used Einstein's theory ( 6 ) to extract Avogadro's number from the slope of the mean-squared particle displacements as a function of time. Perrin's result proved that matter must be discretized at some scale. The random forces imparted by its discrete units - atoms and molecules - give rise to the movement of the particle (Figure 1A).

As a consequence of these random forces, a colloidal particle changes its direction and velocity on time scales smaller than a microsecond. This means that over longer times, it can explore its container (Figure 1B) and - in the language of mechanics - its phase space (momentum and position). If the dispersion is kept free of contaminants that might cause the particles to aggregate, it can reach a state of thermal and chemical equilibrium in perhaps hours or days, reasonable timescales for an experiment.

We can therefore bring the apparatus of statistical thermodynamics to bear on understanding the collective behavior of the particles. In equilibrium at constant temperature, the colloid minimizes its free energy $F=U-T S$, where $S$ is the entropy and $T$ the temperature. The internal energy $U$ is the sum of the kinetic energy, which is constant and can be ignored, and an "effective" potential that accounts for interactions between the solvent molecules and the particle and between the particles themselves (7). If we are given a model or measurement of the effective potential, we can sweep the details of molecules under the rug.

The role of the entropy $S$, and how it relates to the organization of matter, is best illustrated by hard spheres, where the potential energy $U$ is a constant for all configurations. Computer 
simulations in the $1950 \mathrm{~s}(8,9)$ showed that hard spheres spontaneously form a face-centered cubic (FCC) lattice at volume fractions of about 0.50. In 1986, Pusey and van Megen (10) showed that a colloidal dispersion of particles that closely approximated hard spheres showed a fluid-to-crystal transition. Their colloid contained on the order of $10^{14}$ particles, which, in contrast to the simulations, was unambiguously in the thermodynamic limit.

It is perhaps surprising that hard spheres should show a freezing transition. Because the potential energy $U$ is a constant (taken to be zero), we must conclude that the crystallization is driven by an increase in entropy. A simple packing argument (11) shows how. The densest packing of spheres in three dimensions (3D) is the FCC lattice $(12,13)$, with a volume fraction of $74 \%$, whereas the densest disordered packing of spheres occurs at about $64 \%$ volume fraction, as observed in experiments on ball bearings (14). The exact volume fraction of the disordered state depends on the packing protocol (15), but it is always well below the maximum density of the solid. Therefore, at some volume fraction lower than about $64 \%$, a crystalline solid should have higher vibrational entropy than the fluid: if arranged in an FCC lattice at $64 \%$ volume fraction, the particles have room to rattle around their lattice sites, whereas in a disordered arrangement they are jammed (Figure 1C). A more quantitative argument for the phase boundary requires a numerical analysis (16), but the packing argument makes clear that there is an upper bound at which the solid phase must be entropically favored.

The hard-sphere freezing transition shows that entropy should not be thought of as "disorder" but rather as a measure of the configuration space of the particles. The ordering influence of entropy was first predicted in the 1940s by Onsager, who showed that hard rods at high density should orient themselves in the same direction to maximize their freedom to translate (17). This entropic alignment is the origin of the isotropic-nematic transition in a liquid crystal. Since the discovery of hard sphere crystallization, other experiments and simulations on variously shaped hard particles have revealed many entropy-driven transitions from disorder to order (18-21).

The observation that colloids can replicate fundamental phase transitions, as shown in hard spheres (10) and earlier in charged repulsive particles $(22,23)$, has resulted in more than three decades of experiments in which colloids have been used as "model atoms" (24) or "big atoms" (25) for exploring longstanding questions in condensed-matter physics and materials science. Experiments on colloids have examined the formation of defects in crystals under stress $(26,27)$, the dynamics of crystal melting $(28,29)$, the nucleation and growth of crystals $(30,31)$, the role of thermal capillary waves in interfacial phenomena (32), and the glass transition (33). By replacing traditional liquids and crystals by their colloidal counterparts, the experimentalist can image and quantify the motions of individual particles in "real space," as opposed to the less direct "reciprocal space" picture given by scattering techniques. Real-space studies were essential in demonstrating that supercooled liquids showed dynamical heterogeneities $(34,35)$, a precursor to the glass transition. 


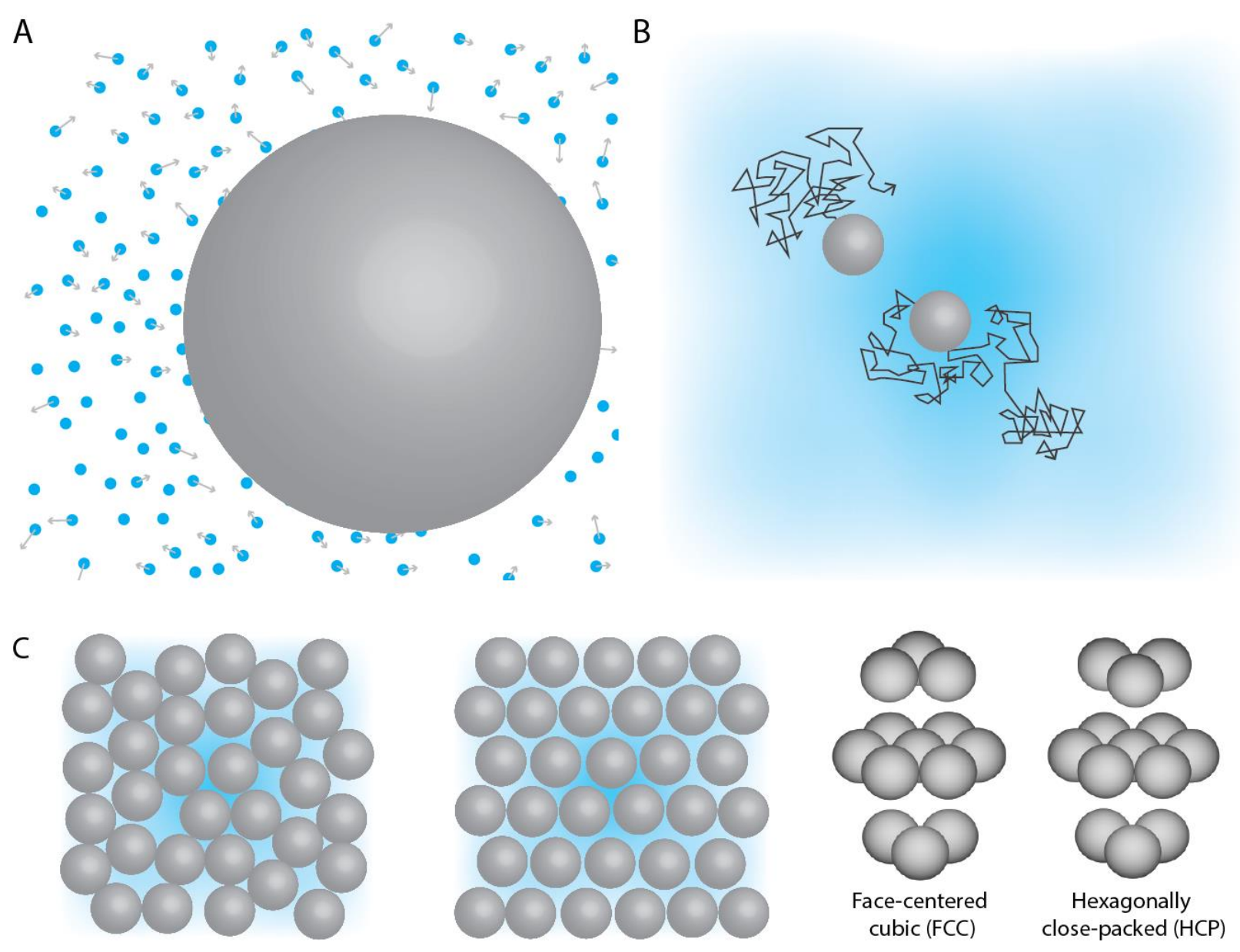

Figure 1. Colloids are a model system for understanding phase transitions. (A) Collisions with solvent molecules (blue) lead to fluctuating, random forces on a particle (gray). (B) On larger time scales, the particles execute a random walk. We can use statistical mechanics to understand the collective behavior because the particles explore their container and interact with each other. (C) Hard spherical particles at a sufficiently high density have less room to move in a fluid phase (left) than in a crystal (right). In 3D, they spontaneously crystallize into a face-centered cubic lattice (right) at 50\% volume fraction. A hexagonally close-packed lattice is shown for contrast. 


\section{From model atoms to colloidal matter}

However, there are differences between colloids and atoms, and these differences result in some of the more interesting features of colloidal matter. The attractions between colloidal particles can be made much shorter ranged (in proportion to the particle size) than those of atoms, and when this is the case, the rates of transitions between structures depend on entropy and hydrodynamics, in contrast to the energy barriers that govern dynamics of atomic systems. Also, because the particles are purely classical objects, vibrational and rotational entropy can stabilize the equilibrium phases, which does not happen in atomic systems at low temperatures.

Ganapathy, Buckley, Gerbode, and Cohen (36) examined how the range of attractive forces affects the growth of crystals. They created colloidal crystal "islands" on a lithographicallypatterned surface by introducing a depletion attraction between the particles. The depletion interaction, which is induced by smaller, non-interacting, nanoscale particles, is fundamentally entropic: when two larger particles come together, they create more space for the smaller particles to move, increasing the total entropy of the system. Although the interaction strength in the Ganapathy experiments was large enough to drive crystallization, the range of the attraction was short, only about $0.5 \%$ of the particle diameter.

The short range of the attraction creates a free-energy barrier for particles to move across the corners and edges of islands. Each particle must break a "bond" (depletion interaction) with one or more particles and then diffuse to reach its new lattice site (Figure 2A). By contrast, in epitaxial growth of atomic crystals, such step-edge barriers arise from the long-ranged interactions between atoms; as the particle crosses the edge, it still "feels" the attractions from next-nearest neighbors in the crystal. Ganapathy et al. found that, surprisingly, the bondbreakage-plus-diffusion process in the colloid leads to growth rates that are quantitatively similar to those in atomic epitaxy. The diffusion process slows down the kinetics, much as the longrange potential barrier does in an atomic system.

However, the similarity between colloidal- and atomic-scale kinetics need not always hold. For a general transition between two states, we must consider how the free energy varies as a function of the configuration of all the particles. Typical models for kinetics in atomic, molecular, and biological systems calculate transition rates from the pathways and barriers between basins on this "free-energy landscape" (37) (Figure 2B). But for colloidal particles this framework breaks down, owing to the short range of the attraction and hydrodynamic drag.

Recent theories of colloidal dynamics take both of these effects into account. Holmes-Cerfon, Gortler, and Brenner (38) considered structural transitions in clusters of spherical particles with short-range attractive interactions. They considered transitions that are conceptually similar to those observed by Ganapathy et al., but generalized: after a bond is broken, particles can diffuse individually or in concert along "soft modes" (Figure 2B). In the limit of an infinitesimally shortranged (or "sticky") attraction - a reasonable approximation for depletion or DNA-mediated (39, 40) attractions - only two parameters, both easily measured in experiment, are needed to predict the transition rates: a diffusion coefficient and the probability of two spheres binding. The rest of the model is pure geometry: the entropy can be calculated as a function of the particle positions along the pathway. Similar models, incorporating not only the effects of drag on individual particles, but also the effects of hydrodynamic interactions between particles, have recently been used to explain non-equilibrium solid-solid transitions in colloidal crystals (41). 
Another key difference between colloidal and atomic matter is that colloidal particles are purely classical objects. If we constrain some of the degrees of freedom, others can stabilize new equilibrium phases. Chen, Bae, and Granick (42) found that colloidal spheres with attractive patches centered on two poles can form a stable Kagome lattice_-an "open" lattice with a low volume fraction (Figure 2C). The Kagome structure is favored over other lattices with equivalent potential energy, some having higher density. Mao, Chen, and Granick (43) later showed that open lattices like the Kagome are favored by the entropy associated with both vibration and rotation of the particles around their lattice sites (Figure 2C). Because these entropic terms can be increased by increasing the patch size, many different phases, including the 3D diamond lattice and even liquid phases (44), might be stabilized by changing patch size and geometry.

This work illustrates the critical role that entropy plays in the organization of colloidal matter, even in the presence of strong attractive interactions. In atomic-scale systems, the vibrational and rotational modes - which in spin systems are better known by the names of their quasi-particles, phonons and magnons - also play a role in determining the equilibrium crystal structures, but these modes can be quantized even at room temperature. In contrast, colloidal systems are purely classical, so there is a continuous spectrum of energies for each mode. No modes can be "frozen out" by reducing the temperature, and all contribute simultaneously to the free energy. 
A

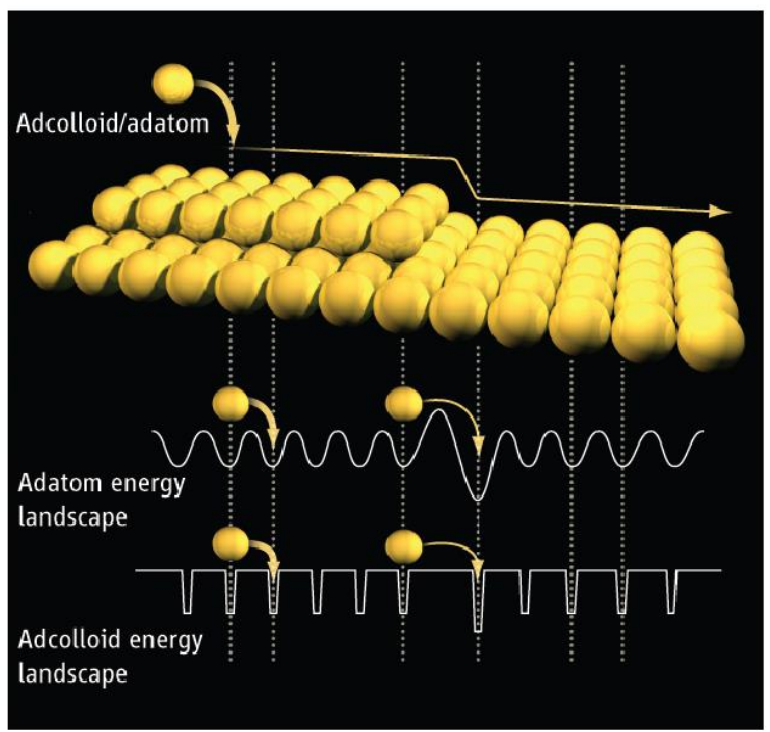

B
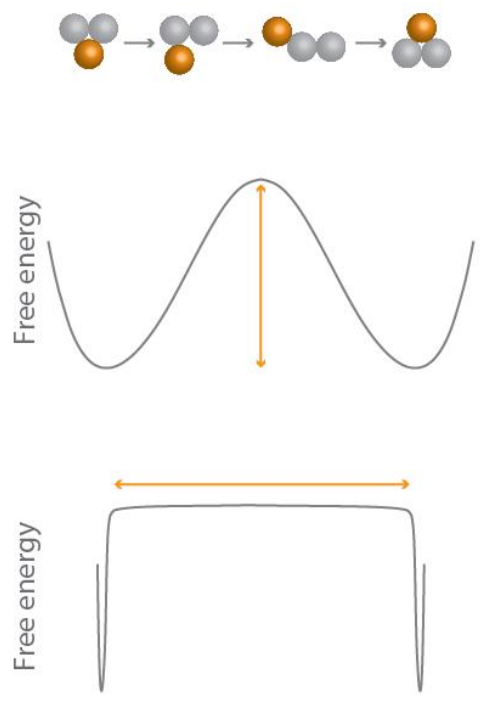

C

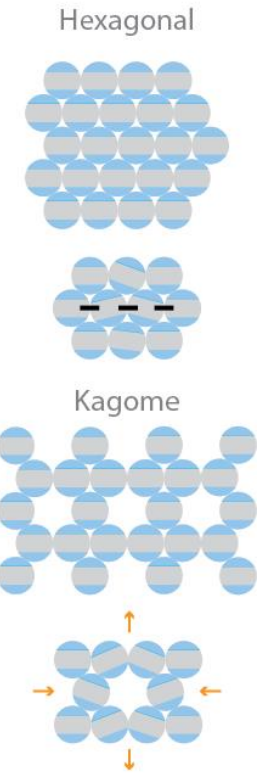

Figure 2. Colloidal matter reveals structural and dynamical features not seen at the atomic scale. (A) Crystals grow through the motion of particles across step-edges (top). Short-range interactions between colloidal particles give rise to a different energy landscape for adcolloids (middle) than for adatoms (bottom). Figure adapted from (105). Reprinted with permission from AAAS. (B) For atoms, the rate of a transition (top) is set by the energy barrier (middle); for colloidal particles, one must consider the rate of diffusion after the bond is broken (bottom). The $\mathrm{x}$-axis indicates the coordinate along the transition path. (C) Janus particles with two attractive patches (blue) assemble into an open Kagome lattice rather than the more dense hexagonal lattice. Both lattices have the same number of contacting patches, but the constraints in the hexagonal lattice (black lines) do not allow the vibrational and rotational degrees of freedom seen in the Kagome (bottom). The short range of the attraction creates a free-energy barrier for particles to move across the corners and edges of islands. Each particle must break a "bond" (depletion interaction) with one or more particles and then diffuse to reach its new lattice site (Figure 2A). By contrast, in epitaxial growth of atomic crystals, such step-edge barriers arise from the long-ranged interactions between atoms; as the particle crosses the edge, it still "feels" the attractions from next-nearest neighbors in the crystal. Ganapathy et al. found that, surprisingly, the bond-breakage-plus-diffusion process in the colloid leads to growth rates that are quantitatively similar to those in atomic epitaxy. The diffusion process slows down the kinetics, much as the long-range potential barrier does in an atomic system. 


\section{Anisotropic and patchy particles}

The "patchy particles" of Chen, Bae, and Granick (42), which interact preferentially along certain directions, are part of a new generation of colloidal particles that break orientational isotropy (45-47). The phase behavior of these particles is potentially much richer than that of spheres. For instance, tetrahedrally-coordinated patchy particles (48) might crystallize into a diamond lattice, and mixtures of patchy particles might form exotic phases such as an "empty liquid" (49), a liquid phase that persists to vanishingly small densities.

For hard particles, changes in shape dramatically alter the equilibrium phases. This point has been examined most thoroughly in simulations. Damasceno et al. (21) explored how the densest equilibrium phase varies as a function of shape in families of convex polyhedra. These polyhedra formed a variety of crystals, liquid crystals, and glasses. In many cases the phase behavior agreed with expectations. For example, nearly spherical polyhedra formed FCC plastic crystals, a configuration that makes intuitive sense: the lattice allows the particles to maximize their vibrational entropy while maintaining freedom to rotate. But other transitions were counterintuitive. Some polyhedra formed more complex phases than expected, and still others failed to crystallize, even though their densest phase is known to be ordered. Although Damasceno et al. were not able to predict the densest equilibrium phase directly from the shape, they were able to correlate the observed phase behavior to two geometrical parameters, the sphericity of the particles and their average coordination number in the fluid phase (Figure 3A). The connection between global phase behavior and local structure was later explained by van Anders et al. (50) in terms of "directional entropic interactions": when two particles orient their faces toward one another, they increase the free volume available to the rest of the particles (Figure 3B). The predictions of Damasceno et al. have not yet been tested in experiments at the colloidal scale, owing to the difficulty of synthesizing hard polyhedra. Inorganic nanoparticles with such shapes have been assembled using depletion interactions or sedimentation $(51,52)$, but experiments on hard colloidal particles would make it possible to visualize the dynamics governing the solid-solid and glass transitions.

An experimentally simpler way to control colloidal assembly is through the topography of the particles. The depletion attraction between two surfaces can be modulated by roughness comparable to the size of the small depleting particles (53). In particles with rough and smooth spots, the smooth parts act as attractive patches. Kraft et al. (54) exploited this effect to assemble analogues of surfactant micelles from sphere doublets comprising one large, rough sphere and one small, smooth sphere (Figure 3C). Another way to control the depletion interaction is to imprint a concave region on an otherwise spherical particle (55). This topographical feature acts as a "lock" for another particle (the "key") having a convex surface that closely matches the contour of the lock. The lock particles can be made by buckling spheres or by growing a spherical particle on a non-spherical seed and then removing the seed (56), leaving a dimple. Such particles can assemble into dimers, chains, and networks (Figure 3D).

All of these colloidal particles, whether spherical or polyhedral, convex or concave, rough or smooth, have a common feature: they are topologically equivalent, or "homeomorphic." In other words, they can all be continuously deformed into one another. It's easiest to understand this if we imagine a colloidal particle as a lump of clay. We can knead it into many different shapes, but unless we punch a hole in it, or roll it into a cylinder and glue the ends together, there is no way to turn it into a torus. So, in terms of topology, even the "non-spherical" particles we have 
seen are spherical! Both the shape and topology of a particle determine the interactions between particles inserted into a medium that is partially ordered, such as a nematic liquid crystal. Defects in the medium can be created by controlling the anchoring conditions or by moving the particles around to make defect lines and even knots $(57)$. Smalyukh and coworkers $(58,59)$ showed that new classes of defect structures could be stabilized by particles with "handles" (Figure 3D) or knots (59). Handles are a topological feature: a sphere has none, a ring or coffee mug one, and two or more rings glued together in the plane have multiple handles. Ultimately, these topologically distinct particles may be able to self-assemble into novel structures, owing to the effective interactions that emerge as the liquid crystal minimizes the elastic energy associated with the defects $(60,61)$. 

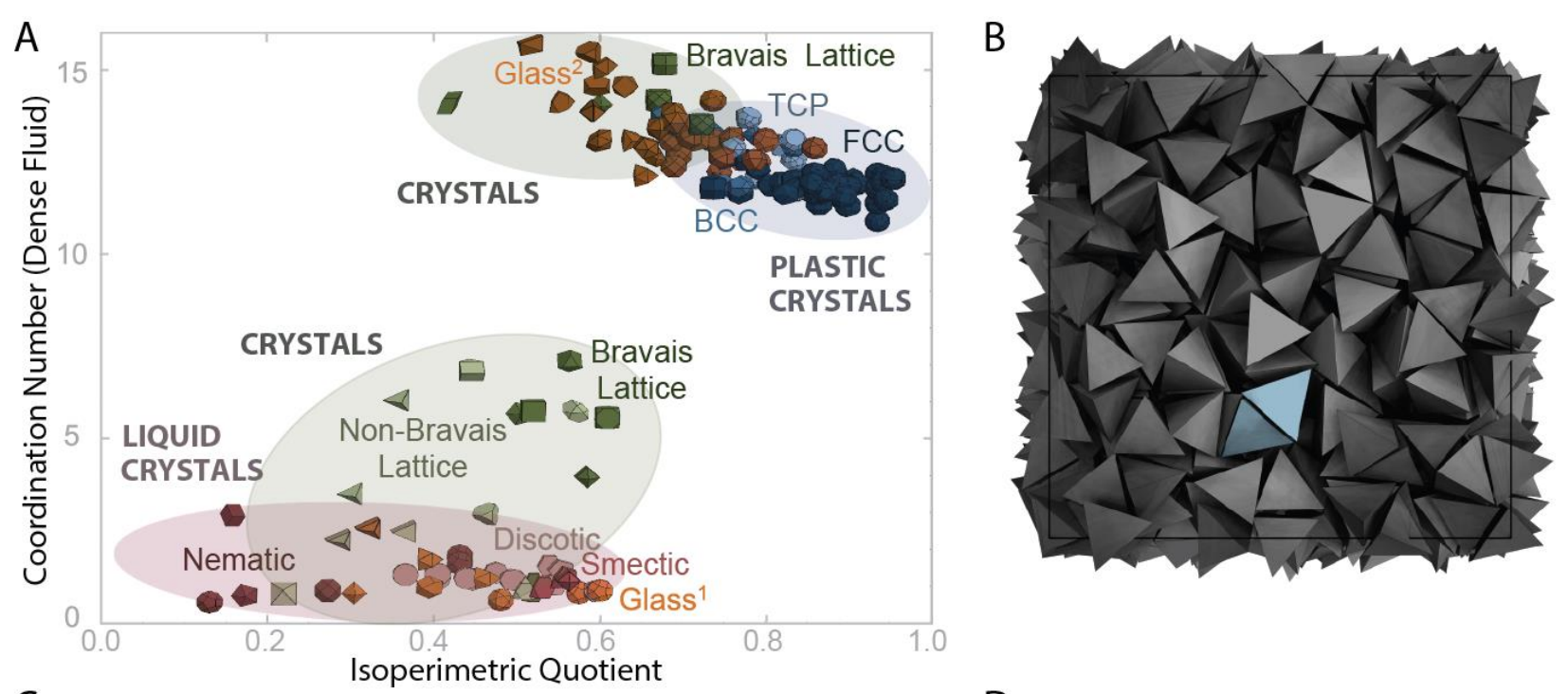

C
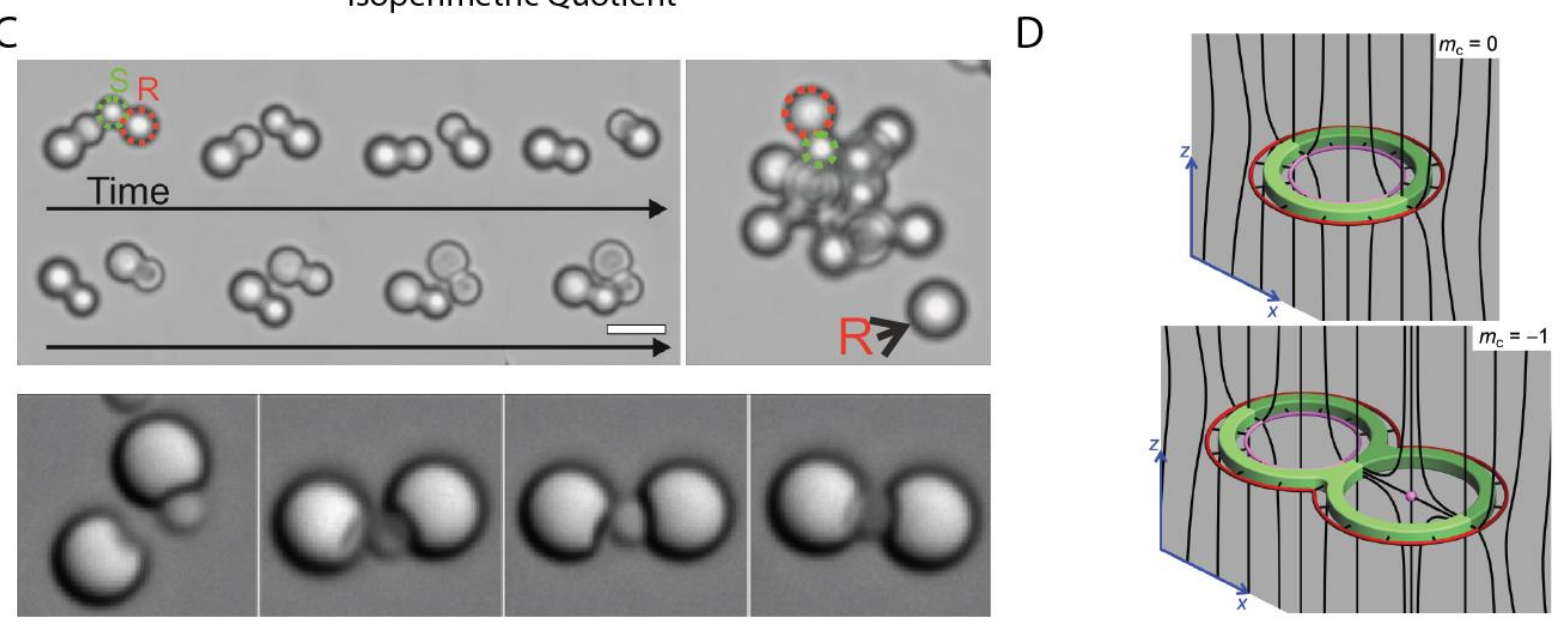

Figure 3. The shape, topography, and topology of nonspherical particles can be used to control assembly. (A) Equilibrium phases found in simulations of hard convex polyhedral correlate with the coordination number in the fluid and the degree of sphericity of the particles (adapted from (21). Reprinted with permission from AAAS). (B) To maximize the entropy of the system, the particles tend to orient with their faces parallel and close to one another. The tendency can be viewed as a "directional entropic force" (adapted from (50)). (C) Topographical features control assembly in the presence of depletion forces. Top, time series of micrographs showing dimers of rough ("R") and smooth ("S") particles assembling into analogues of micelles. Scale bar, $5 \mu \mathrm{m}$ (adapted from (54)). Bottom, time series of micrographs showing 2- $\mu$ m-diameter particles with concave dimples attracting convex particles and assembling into chains (Adapted by permission from Macmillan Publishers Ltd: ref. (55), copyright 2010). (D) Particles with different numbers of topological handles affect the director field (black lines) in a liquid crystal. The interactions between such particles can be controlled by the anchoring conditions and the number of handles (Adapted by permission from Macmillan Publishers Ltd: ref.(58), copyright 2013). 


\section{Colloidal matter on curved surfaces}

Geometry allows us to rationalize, and in some cases predict, how colloidal matter organizes, and even how its dynamics emerge. But what if we could change the rules of geometry itself?

We can do this by curving the space that the colloid inhabits. Imagine we put hard spheres on a spherical surface. At high density, the system favors a triangular lattice, which corresponds to the densest packing of spheres in 2D (62). Each particle in the lattice has six nearest neighbors arranged in a hexagon, so that each component triangle of the hexagon subtends an angle of $\pi / 3$. But on a sphere, the sum of the interior angles of a triangle must exceed $\pi$ (Figure 4A), which means that the hexagon is strained.

How does the system accommodate this curvature-induced strain? Part of the answer comes from mathematics. We can break down any 2D arrangement of points on the sphere into a network of (not necessarily regular) triangles that form a closed polyhedron. A famous result from Euler relates the number of vertices $V$, edges $E$, and faces $F$ of the polyhedron: $V-E+F=2$. The number 2, called the Euler characteristic, indicates that some particles must have fewer than 6 nearest neighbors; otherwise, we would have $V-E+F=0$.

The simplest way to satisfy Euler's theorem is to add 12 defects in which a particle is coordinated by five, rather than six, nearest neighbors. These 12 five-fold disclinations, as they are called, can be seen in an old-style, hand-stitched soccer ball (or a "football" as it is known in most places), which contains exactly 12 pentagonal panels, the rest being hexagonal. The 12 pentagons allow the otherwise hexagonal tiling to make up for the angular surplus imposed by the Gaussian curvature. More generally, we can assign each five-fold disclination a topological "charge" of +1 , and each seven-fold defect a charge of -1 . The total disclination charge must be six times the Euler characteristic, which varies for different topologies.

Geometry doesn't entirely dictate the structure, though. The potential between the particles also matters (63). Bausch et al. (64) observed nearly hard-sphere colloidal particles bound to the surface of a spherical droplet and found that although the total disclination charge was exactly 12, in agreement with Euler's theorem, there were more than 12 defects. The excess defects consisted of pairs of five-fold and seven-fold disclinations aligned in chains that Bausch et al. termed "grain boundary scars" (Figure 4B). They argued that the scars minimize the elastic energy associated with the required disclinations (note that in theoretical models of the free energy, one treats the crystal as a continuous elastic medium and then calculates the deformation arising from the curvature-induced strain and the defects). The elastic energy implicitly includes an entropic contribution; indeed, for hard spheres, this is the only contribution. Bausch et al.'s elastic model showed that a single isolated five-fold disclination would incur a large local elastic strain, resulting in a large elastic energy penalty. To avoid the penalty, the crystal creates chains of defects, which gradually reduce the strain while keeping the total disclination charge constant.

Different defect motifs emerge to accommodate gradients in curvature like those found in a capillary bridge. Topologically such bridges are equivalent to cylinders, which have an Euler characteristic of zero-meaning that no defects are required. The curvature, however, can vary across the bridge: it is zero near the top or bottom plates, and positive or negative at the center, depending on the distance between the plates. Irvine, Vitelli, and Chaikin (65) examined defects in a colloidal suspension confined to a capillary bridge and found that as they pulled the surfaces apart, chains of dislocations formed with their seven-fold disclinations closer to the center of the 
bridge (Figure 4C). The total disclination charge remained zero, as required by topology. These polarized dislocations allow the crystal to minimize the elastic energy associated with the gradient in curvature, much as pleats allow a pair of trousers to accommodate the different curvatures of the wearer's waist and hips.

More recent experimental work has examined how curvature can affect the mobility of defects (66) and the growth of crystals (67). Meng and coworkers (67) found that particles with shortranged depletion interactions on a spherical droplet formed crystals consisting of connected "ribbons," anisotropic domains with widths much smaller than their lengths (Figure 4D). The ribbon-like growth is a consequence of elastic considerations. When the attraction between particles is short-ranged, the crystal is brittle, and trying to wrap it around the sphere is like trying to gift-wrap a basketball: the wrapping paper can't stretch, and if we are not allowed to wrinkle it, our only option is to tear it into strips. Multiple strips of the same width allow the crystal to cover a large area with minimal strain.

We still do not have a full picture of how curvature and the interparticle potential affect the kinetics of phase transitions. Simulations $(68,69)$ of crystal growth on curved surfaces usually employ long-range attractive potentials, which give rise to dislocations, grain boundary scars, or pleats in the growing crystal, depending on the curvature and its gradient. But in colloidal matter, where attractions are short-ranged, it could well be difficult to reach the ground states, which must (by Euler's theorem) include topological defects. It will therefore be interesting to see if biological systems on curved surfaces, such viral capsids and lipid vesicles, follow the same growth pattern shown by colloidal crystals on curved surfaces. 
A
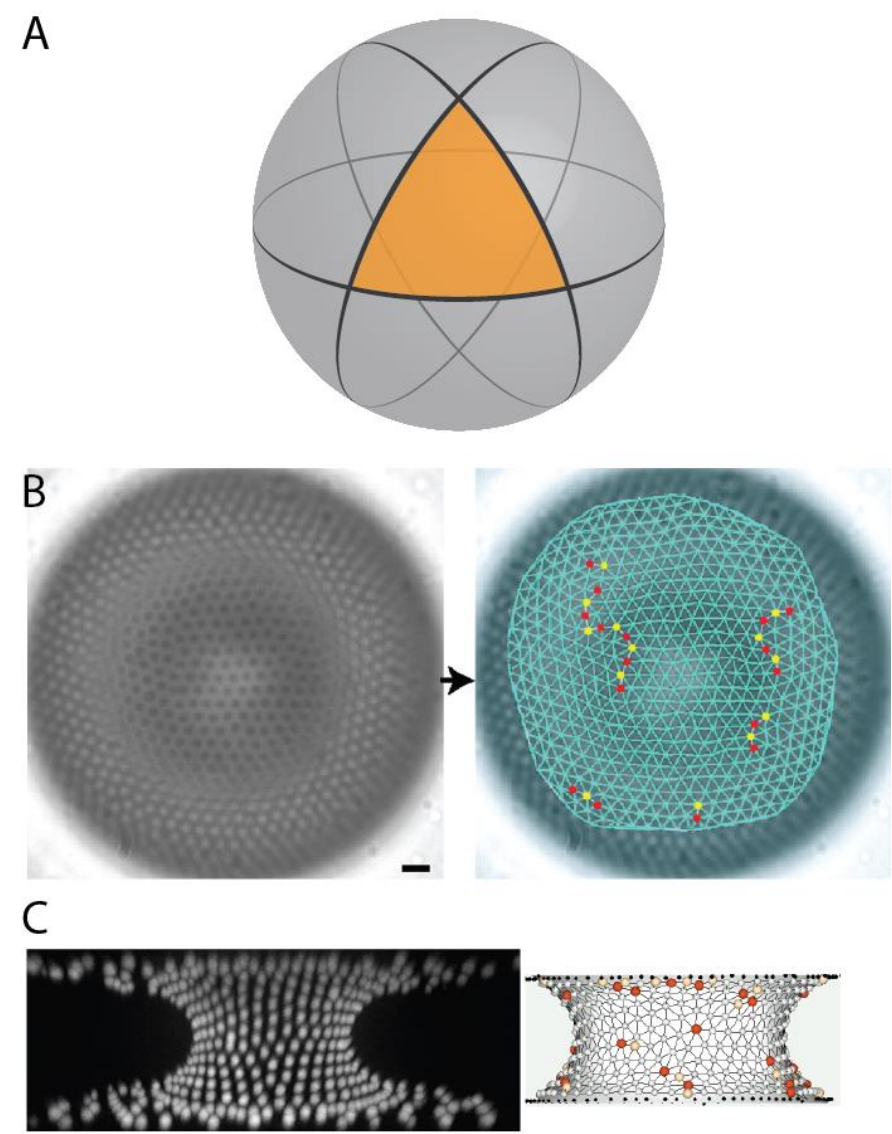

D

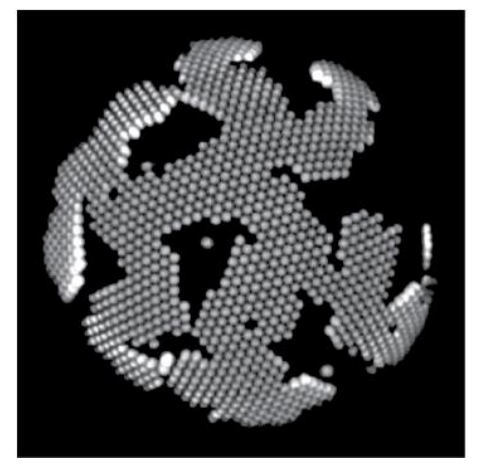

Figure 4. Curvature controls defects and growth in colloidal crystals. (A) The sum of the interior angles of an equilateral triangle on a sphere must exceed $\pi$. As a consequence, a hexagonal lattice on the sphere is strained. (B) To minimize the elastic strain caused by the curvature, hardsphere crystals on a spherical surface (left) form chains of disclinations known as grain-boundary scars (right; red: five-fold disclination; yellow: seven-fold disclination). From (64). Reprinted with permission from AAAS. (C) Polarized disclination pairs form in crystals on a capillary bridge (left). These "pleats" minimize the elastic strain associated with the gradient in curvature (yellow: five-fold disclination; red: seven-fold disclination). Adapted by permission from Macmillan Publishers Ltd: ref. (65), copyright 2010. (D) Attractive particles on a sphere form ribbon-like domains, which allow the crystal to minimize its elastic energy while still covering a large surface area (image from Guangnan Meng). 


\section{Geometrical frustration in three dimensions}

Taken together, the results of the previous section show that curvature "frustrates" the growth of 2D colloidal crystals of spherical particles. It makes the locally-favored packing of particles incompatible with the space-filling packing, resulting in disorder. Theories of geometrical frustration reason in the opposite direction to understand 3D disorder: given a particular 3D system, can we understand its propensity for disorder in terms of a competition between locally and globally favorable structures?

There are many 3D disordered systems; indeed, most colloids form glasses or gels. The origins of these non-equilibrium phases, whether in colloidal matter or condensed matter as a whole, remain poorly understood. One body of work $(70,71)$ attempts to connect the slowdown of dynamics near the glass or gel transition to a growing structural length scale, perhaps associated with locally-favored structures.

The challenge, then, is to determine the locally-favored structures. This problem has a long history in materials science and condensed-matter physics, beginning with a 1952 paper by Frank (72) showing that an icosahedron of 13 spheres interacting through a Lennard-Jones 6-12 potential has an $8.4 \%$ lower energy than arrangements that occur in the FCC or hexagonally close-packed (HCP) lattices (Figure 5A). Frank hypothesized that these stable icosahedral arrangements might be common in liquids and, furthermore, that they might allow certain liquids to be supercooled well below their freezing point, since the five-fold symmetry of icosahedra is incompatible with crystalline order. Later calculations found that five-fold symmetric structures, though not necessarily icosahedra, are more stable than lattice-like clusters for other model potentials and for larger numbers of spheres (73). The statistical mechanics of such finite systems has become a field of its own $(37,74)$. In the last decade Frank's hypothesis was verified by X-ray experiments that revealed structures with five-fold symmetry in metallic liquids (75).

However, we must be careful when applying Frank's hypothesis to colloidal matter. The Lennard-Jones 6-12 and related potentials are reasonable approximations for certain nanoparticles (76) but are poor models for colloids, which have much shorter-range attractions. Moreover, entropy must play a role in structuring colloids, even when the potential is attractive.

In hard spheres, where the only structuring influence is entropy, the locally-favored structures do not appear to be icosahedra, except when the particles are confined (77). Colloidal hard spheres in 3D show a glass transition at a volume fraction of about 0.60 or higher, depending on the polydispersity (78). "Supercooled" fluids of such particles - that is, dispersions that remain fluid although they have been concentrated to volume fractions above the freezing transition-show structural and dynamical features similar to atomic-scale liquids (79). Yet experiments show that the local structure of supercooled colloidal fluids differs qualitatively from that of metallic liquids. Gasser et al. found few true icosahedra in the bulk of supercooled hard-sphere fluids (80). Simulations by Royall, Malins, Dunleavy, and Pinney at larger supercooling (higher volume fraction) also did not find icosahedra to be favored, although 10-membered clusters that are fragments of icosahedra were a common motif (81). However, the same study suggests that even deeper supercooling is necessary to resolve the connection between locally-favored structures and the glass transition.

Experiments on random packings of hard macroscopic spheres, beginning with Stephen Hales's examination of pea packings in 1727 (82), and continuing to Bernal and Finney's studies of ball 
bearing packings $(14,83)$ and more recent work on x-ray tomography of spherical beads $(84)$, have found five-fold local packings but also many other local structures that are incompatible with crystalline order. As van Meel and coworkers noted (85) "Bernal and many after him have indeed observed just about any small polyhedron in hard-sphere fluids." It is still not clear which locally-favored structures we should be looking for in hard-sphere fluids.

When the particles attract one another, locally-favored structures can be found using energetic calculations or cluster lifetimes (86). Royall et al. (87) found that icosahedral, deltahedral, and bipyramidal clusters minimize the potential energy of a Morse potential with a short range parameter, which approximates a depletion interaction between spherical particles. Using confocal microscopy, they looked for these clusters inside a colloidal gel and found that they occurred in long-lived percolating networks (Figure 5B). These networks might contribute to the slowdown of dynamics near the gel transition.

The stability of such clusters is also influenced by entropy $(88,89)$. One entropic effect that must be considered is the huge number of competing structures at any given cluster size: for example, for a 10-sphere cluster, there are nearly 400 potential-energy minima, most of which are degenerate $(90,91)$. A second entropic effect involves the number of ways each cluster can form. Meng et al. found that clusters of attractive spherical particles formed asymmetric structures in equilibrium (89). This tendency, which arises because lower-symmetry clusters have more permutations of particles that result in the same structure, strongly selects against the icosahedron and five-fold symmetry, while favoring polytetrahedral clusters (Figure 5C). This case shows yet another way in which short-range attractions and entropy in colloidal matter conspire to produce results different from (and, in this case, almost exactly the opposite of) atomic systems.

There is still no direct correlation between the stability of certain clusters and a growing structural length scale in arrested colloidal phases. But perhaps this has more to do with the system than the model (92). Spherical particles may be one of the simpler experimental systems to examine, but they are also one of the trickiest to think about geometrically. Thus some experiments have begun to examine slow dynamics in dispersions of anisotropic particles (93).

Hard tetrahedra may be a better system for testing theories of geometrical frustration because they "prefer" higher dimensions: although they cannot fill 3D Euclidean space, they can perfectly tile the surface of a four-dimensional hypersphere (94). Just as putting hard spheres on the surface of a sphere leads to disclinations, so does putting densely packed tetrahedra in flat (Euclidean) space $(95,96)$; both systems are subject to a curvature-induced elastic strain. Simulations have found that hard tetrahedra form a disordered, quasicrystal phase that persists to high densities, even though a denser ordered phase can form (97). It is interesting to consider whether the stability of this quasicrystal might be traced to locally-favored structures that - like the ribbons we saw in crystals of attractive spheres on a sphere (67) - minimize the curvatureinduced elastic strain. 


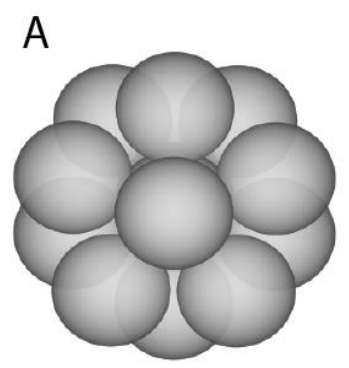

Icosahedron

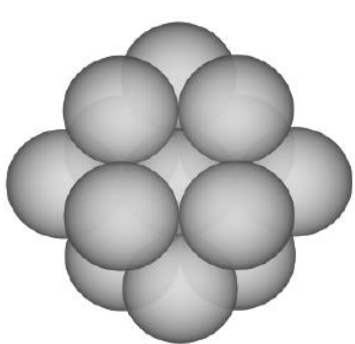

FCC

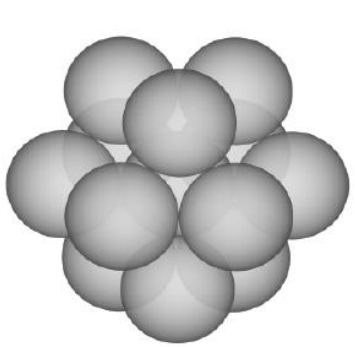

$\mathrm{HCP}$
C
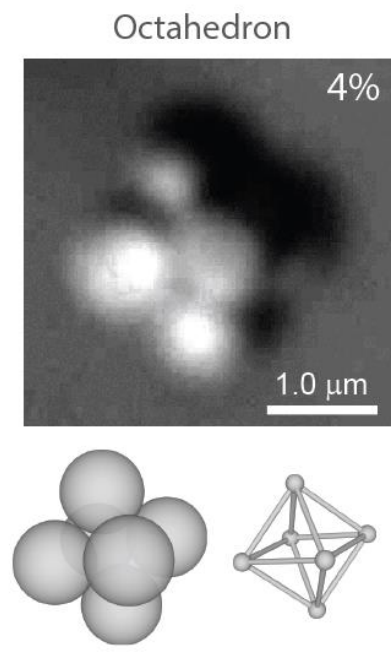

Polytetrahedron
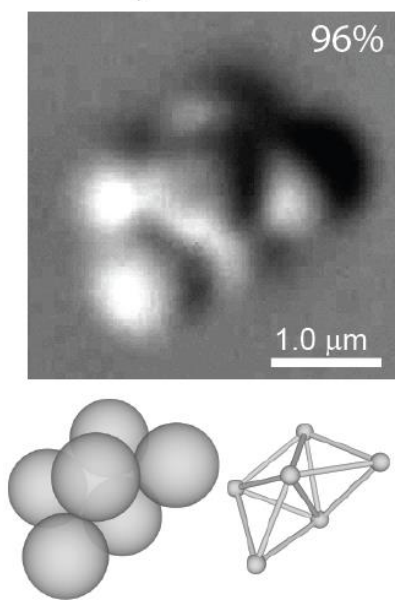

Figure 5. The tension between locally and globally favored structures leads to frustration. (A) The lowest potential-energy configuration of 13 spheres interacting through a Lennard-Jones potential is an icosahedron. Unlike the FCC or HCP clusters, icosahedra cannot tile space. (B) Particles interacting through short-range attractions form a gel. The minimal-energy clusters of such particles (shown in colors) form a percolating network (Adapted by permission from Macmillan Publishers Ltd: ref. (87), copyright 2008). (C) Entropy favors asymmetry in local structure. Six particles with short-range attractions form an asymmetric configuration (the polytetrahedron) more than 24 times as often as they form a symmetric structure (the octahedron) in equilibrium. Both structures have the same potential energy. The difference in probabilities is primarily due to the higher rotational entropy for the asymmetric structure. 


\section{Looking forward}

Perhaps it should not be surprising that geometry and packing arguments can be used to understand the organization of colloidal matter. The earliest incarnations of colloidal particles were nanoscale objects with haphazard arrangements of charges and uncontrolled polydispersity. Advances in synthesis and fabrication have transformed colloids into their modern form: highly symmetric shapes with well-controlled size distributions, surface chemistry, topography, and topology. In other words, colloidal matter is designed to obey geometrical rules.

What is surprising is the richness of the phenomena that emerge when we combine these wellcontrolled particles with simple constraints. These phenomena occur because thermodynamic quantities such as the elastic energy depend sensitively on the interaction potential, and the entropy on how the particles are allowed to fluctuate. When we constrain the dominant degrees of freedom (by, for example, introducing patchy attractions), the other degrees of freedom that we previously neglected now come to the fore, stabilizing new structures.

In most cases, however, geometrical arguments remain post-hoc explanations for the collective behavior we discover in experiment or simulation. Steps toward predictive frameworks have been made in the geometrical model of the free-energy landscape of Holmes-Cerfon et al. (38) and the directional entropic forces of van Anders et al. (50). But there are many other transitions and structural motifs that remain to be discovered in the vast parameter space now accessible to the experimentalist — a space spanned by shape, topography, topology, and curvature.

Dynamics offers yet another dimension of control, one which is difficult to vary in atomic systems but in colloidal matter emerges naturally from the interactions between particles and fluid. Even at the single particle level, the thermally-driven motion of nonspherical particles (98) and of particles in anisotropic fluids (99) continues to reveal surprises, nearly 100 years after Perrin's experiments. The hydrodynamic interactions between these particles can not only select for certain phases (41), but can also result in different collective phenomena when the driving forces are intrinsic or extrinsic energy sources rather than thermal fluctuations (100). Such active colloidal systems show nonequilibrium self-organization with complexity rivaling that of biological systems such as living cells, bacterial baths, and animal flocks.

Another variable is specificity. DNA-functionalized colloidal particles have effective attractions that are both short-ranged and specific (101), and each particle can carry more than 40 different strands of DNA (102). It is therefore possible to explore systems in which the number of independent pair interactions is comparable to the number of particles. Collective effects should emerge from the interplay between specificity and packing constraints in such "polygamous" systems, which have no equivalent at the molecular scale.

Understanding the effects of specificity, dynamics, and the many other dimensions of colloidal matter will require new experiments and theoretical models. But with such understanding we might resolve longstanding challenges, such as the "inverse" problem of creating a colloidal system that will self-assemble into a prescribed structure. Optical materials are a prime application, since the scale of colloidal particles is on the order of visible light. The optimal structures for materials that show structural colors (103) and photonic band gaps (104) are not crystals, but disordered materials with short-range correlations. Thus, the framework of geometrical frustration, which has been used to explain disordered systems, could be used to design new ones instead, through specific choices for the shapes or interactions of the particles. 
Future discoveries in colloidal matter will continue to come from systematic and iterative collaborations between those who synthesize particles, those who measure their structure and dynamics, and those who construct models explaining the phenomena. Colloidal matter is, and will continue to be, where geometry, chemistry, and physics meet.

\section{References and Notes:}

1. In another semantic shift, the word "colloid" is increasingly used to refer to an individual colloidal particle. In this article I use the traditional definition, where "colloid" means "colloidal dispersion."

2. F. Li, D. P. Josephson, A. Stein, Colloidal Assembly: The Road from Particles to Colloidal Molecules and Crystals. Angew. Chem. Int. Ed. 50, 360-388 (2011).

3. O. Kruglova, P.-J. Demeyer, K. Zhong, Y. Zhou, K. Clays, Wonders of colloidal assembly. Soft Matter. 9, 9072-9087 (2013).

4. R. Brown, A brief account of microscopical observations made in the months of June, July and August 1827, on the particles contained in the pollen of plants; and on the general existence of active molecules in organic and inorganic bodies. Philos. Mag. Ser. 2. 4, 161173 (1828).

5. J. Perrin, Mouvement brownien et réalité moléculaire. Ann. Chim. Phys. 18, 5-104 (1909).

6. A. Einstein, Über die von der molekularkinetischen Theorie der Wärme geforderte Bewegung von in ruhenden Flüssigkeiten suspendierten Teilchen. Ann. Phys. 322, 549-560 (1905).

7. D. Frenkel, in Soft Condensed Matter Physics in Molecular and Cell Biology, W. C. K. Poon, D. Andelman, Eds. (CRC Press, 2006), Scottish Graduate Series, pp. 19-48.

8. W. W. Wood, J. D. Jacobson, Preliminary Results from a Recalculation of the Monte Carlo Equation of State of Hard Spheres. J. Chem. Phys. 27, 1207-1208 (1957).

9. B. J. Alder, T. E. Wainwright, Phase Transition for a Hard Sphere System. J. Chem. Phys. 27, 1208-1209 (1957).

10. P. N. Pusey, W. van Megen, Phase behaviour of concentrated suspensions of nearly hard colloidal spheres. Nature. 320, 340-342 (1986).

11. T. C. Lubensky, Soft Condensed Matter Physics. Solid State Commun. 102, 187-197 (1997).

12. T. C. Hales, Sphere packings, I. Discrete Comput. Geom. 17, 1-51 (1997).

13. T. C. Hales, Sphere Packings, II. Discrete Comput. Geom. 18, 135-149 (1997). 
14. J. D. Bernal, J. Mason, Packing of Spheres: Co-ordination of Randomly Packed Spheres. Nature. 188, 910-911 (1960).

15. S. Torquato, T. M. Truskett, P. G. Debenedetti, Is Random Close Packing of Spheres Well Defined? Phys. Rev. Lett. 84, 2064-2067 (2000).

16. A. D. Dinsmore, A. G. Yodh, D. J. Pine, Phase diagrams of nearly-hard-sphere binary colloids. Phys. Rev. E. 52, 4045-4057 (1995).

17. L. Onsager, The Effects of Shape on the Interaction of Colloidal Particles. Ann. N. Y. Acad. Sci. 51, 627-659 (1949).

18. D. Frenkel, H. N. W. Lekkerkerker, A. Stroobants, Thermodynamic stability of a smectic phase in a system of hard rods. Nature. 332, 822-823 (1988).

19. D. Frenkel, Entropy-driven phase transitions. Phys. Stat. Mech. Its Appl. 263, $26-38$ (1999).

20. Z. Dogic, S. Fraden, Ordered phases of filamentous viruses. Curr. Opin. Colloid Interface Sci. 11, 47-55 (2006).

21. P. F. Damasceno, M. Engel, S. C. Glotzer, Predictive Self-Assembly of Polyhedra into Complex Structures. Science. 337, 453-457 (2012).

22. D. W. Schaefer, B. J. Ackerson, Melting of Colloidal Crystals. Phys. Rev. Lett. 35, $1448-$ 1451 (1975).

23. H. M. Lindsay, P. M. Chaikin, Elastic properties of colloidal crystals and glasses. J. Chem. Phys. 76, 3774-3781 (1982).

24. V. J. Anderson, H. N. W. Lekkerkerker, Insights into phase transition kinetics from colloid science. Nature. 416, 811-815 (2002).

25. W. Poon, Colloids as Big Atoms. Science. 304, 830-831 (2004).

26. P. Schall, I. Cohen, D. A. Weitz, F. Spaepen, Visualization of Dislocation Dynamics in Colloidal Crystals. Science. 305, 1944-1948 (2004).

27. P. Schall, I. Cohen, D. A. Weitz, F. Spaepen, Visualizing dislocation nucleation by indenting colloidal crystals. Nature. 440, 319-323 (2006).

28. A. M. Alsayed, M. F. Islam, J. Zhang, P. J. Collings, A. G. Yodh, Premelting at Defects Within Bulk Colloidal Crystals. Science. 309, 1207-1210 (2005).

29. Z. Wang, F. Wang, Y. Peng, Z. Zheng, Y. Han, Imaging the Homogeneous Nucleation During the Melting of Superheated Colloidal Crystals. Science. 338, 87-90 (2012).

30. U. Gasser, E. R. Weeks, A. Schofield, P. N. Pusey, D. A. Weitz, Real-Space Imaging of Nucleation and Growth in Colloidal Crystallization. Science. 292, 258-262 (2001). 
31. J. Russo, H. Tanaka, The microscopic pathway to crystallization in supercooled liquids. Sci. Rep. 2 (2012), doi:10.1038/srep00505.

32. D. G. A. L. Aarts, M. Schmidt, H. N. W. Lekkerkerker, Direct Visual Observation of Thermal Capillary Waves. Science. 304, 847-850 (2004).

33. P. N. Pusey, W. van Megen, Observation of a glass transition in suspensions of spherical colloidal particles. Phys. Rev. Lett. 59, 2083-2086 (1987).

34. W. K. Kegel, A. van Blaaderen, Direct Observation of Dynamical Heterogeneities in Colloidal Hard-Sphere Suspensions. Science. 287, 290-293 (2000).

35. E. R. Weeks, J. C. Crocker, A. C. Levitt, A. Schofield, D. A. Weitz, Three-Dimensional Direct Imaging of Structural Relaxation Near the Colloidal Glass Transition. Science. 287, 627-631 (2000).

36. R. Ganapathy, M. R. Buckley, S. J. Gerbode, I. Cohen, Direct Measurements of Island Growth and Step-Edge Barriers in Colloidal Epitaxy. Science. 327, 445-448 (2010).

37. D. Wales, Energy Landscapes: Applications to Clusters, Biomolecules and Glasses (Cambridge University Press, 2004), Cambridge Molecular Science.

38. M. Holmes-Cerfon, S. J. Gortler, M. P. Brenner, A geometrical approach to computing free energy landscapes from short-ranged potentials. Proc. Natl. Acad. Sci. 110, E5-E14 (2013).

39. W. B. Rogers, J. C. Crocker, Direct measurements of DNA-mediated colloidal interactions and their quantitative modeling. Proc. Natl. Acad. Sci. 108, 15687 -15692 (2011).

40. P. Varilly, S. Angioletti-Uberti, B. M. Mognetti, D. Frenkel, A general theory of DNAmediated and other valence-limited colloidal interactions. J. Chem. Phys. 137, 094108094108-15 (2012).

41. I. C. Jenkins, M. T. Casey, J. T. McGinley, J. C. Crocker, T. Sinno, Hydrodynamics selects the pathway for displacive transformations in DNA-linked colloidal crystallites. Proc. Natl. Acad. Sci. 111, 4803-4808 (2014).

42. Q. Chen, S. C. Bae, S. Granick, Directed self-assembly of a colloidal kagome lattice. Nature. 469, 381-384 (2011).

43. X. Mao, Q. Chen, S. Granick, Entropy favours open colloidal lattices. Nat. Mater. 12, $217-$ 222 (2013).

44. F. Smallenburg, F. Sciortino, Liquids more stable than crystals in particles with limited valence and flexible bonds. Nat. Phys. 9, 554-558 (2013).

45. S. C. Glotzer, M. J. Solomon, Anisotropy of building blocks and their assembly into complex structures. Nat. Mater. 6, 557-562 (2007). 
46. E. Duguet, A. Désert, A. Perro, S. Ravaine, Design and elaboration of colloidal molecules: an overview. Chem. Soc. Rev. 40, 941 (2011).

47. S. Sacanna, D. J. Pine, G.-R. Yi, Engineering shape: the novel geometries of colloidal selfassembly. Soft Matter. 9, 8096-8106 (2013).

48. Y. Wang et al., Colloids with valence and specific directional bonding. Nature. 491, 51-55 (2012).

49. E. Bianchi, J. Largo, P. Tartaglia, E. Zaccarelli, F. Sciortino, Phase Diagram of Patchy Colloids: Towards Empty Liquids. Phys. Rev. Lett. 97, 168301 (2006).

50. G. van Anders, D. Klotsa, N. K. Ahmed, M. Engel, S. C. Glotzer, Understanding shape entropy through local dense packing. Proc. Natl. Acad. Sci. 111, E4812-E4821 (2014).

51. K. L. Young et al., A Directional Entropic Force Approach to Assemble Anisotropic Nanoparticles into Superlattices. Angew. Chem. Int. Ed. 52, 13980-13984 (2013).

52. J. Henzie, S. C. Andrews, X. Y. Ling, Z. Li, P. Yang, Oriented assembly of polyhedral plasmonic nanoparticle clusters. Proc. Natl. Acad. Sci. 110, 6640-6645 (2013).

53. K. Zhao, T. G. Mason, Directing Colloidal Self-Assembly through Roughness-Controlled Depletion Attractions. Phys. Rev. Lett. 99, 268301 (2007).

54. D. J. Kraft et al., Surface roughness directed self-assembly of patchy particles into colloidal micelles. Proc. Natl. Acad. Sci. 109, 10787-10792 (2012).

55. S. Sacanna, W. T. M. Irvine, P. M. Chaikin, D. J. Pine, Lock and key colloids. Nature. 464, $575(2010)$.

56. S. Sacanna et al., Shaping colloids for self-assembly. Nat. Commun. 4, 1688 (2013).

57. U. Tkalec, M. Ravnik, S. Čopar, S. Žumer, I. Muševič, Reconfigurable Knots and Links in Chiral Nematic Colloids. Science. 333, 62-65 (2011).

58. B. Senyuk et al., Topological colloids. Nature. 493, 200-205 (2013).

59. A. Martinez et al., Mutually tangled colloidal knots and induced defect loops in nematic fields. Nat. Mater. 13, 258-263 (2014).

60. P. Poulin, H. Stark, T. C. Lubensky, D. A. Weitz, Novel Colloidal Interactions in Anisotropic Fluids. Science. 275, 1770-1773 (1997).

61. I. Muševič, Nematic colloids, topology and photonics. Philos. Trans. R. Soc. Math. Phys. Eng. Sci. 371, 20120266 (2013).

62. A. Thue, Om nogle geometrisk-taltheoretiske Theoremer. Forh Ved Skand. Naturforskeres, 352-353 (1892). 
63. M. J. Bowick, L. Giomi, Two-dimensional matter: order, curvature and defects. Adv. Phys. 58, 449-563 (2009).

64. A. R. Bausch et al., Grain Boundary Scars and Spherical Crystallography. Science. 299, 1716-1718 (2003).

65. W. T. M. Irvine, V. Vitelli, P. M. Chaikin, Pleats in crystals on curved surfaces. Nature. 468, 947-951 (2010).

66. W. T. M. Irvine, M. J. Bowick, P. M. Chaikin, Fractionalization of interstitials in curved colloidal crystals. Nat. Mater. 11, 948-951 (2012).

67. G. Meng, J. Paulose, D. R. Nelson, V. N. Manoharan, Elastic Instability of a Crystal Growing on a Curved Surface. Science. 343, 634-637 (2014).

68. R. Backofen, A. Voigt, T. Witkowski, Particles on curved surfaces: A dynamic approach by a phase-field-crystal model. Phys. Rev. E. 81, 025701 (2010).

69. A. Luque, D. Reguera, A. Morozov, J. Rudnick, R. Bruinsma, Physics of shell assembly: Line tension, hole implosion, and closure catastrophe. J. Chem. Phys. 136, 184507 (2012).

70. S. Karmakar, C. Dasgupta, S. Sastry, Growing Length Scales and Their Relation to Timescales in Glass-Forming Liquids. Annu. Rev. Condens. Matter Phys. 5, 255-284 (2014).

71. C. P. Royall, S. R. Williams, The role of local structure in dynamical arrest. ArXiv14055691 Cond-Mat (2014) (available at http://arxiv.org/abs/1405.5691).

72. F. C. Frank, Supercooling of Liquids. Proc. R. Soc. Lond. Ser. Math. Phys. Sci. 215, 43-46 (1952).

73. J. P. K. Doye, D. J. Wales, Structural consequences of the range of the interatomic potential - A menagerie of clusters. J. Chem. Soc. Faraday Trans. 93, 4233-4243 (1997).

74. F. H. Stillinger, T. A. Weber, Packing Structures and Transitions in Liquids and Solids. Science. 225, 983-989 (1984).

75. H. Reichert et al., Observation of five-fold local symmetry in liquid lead. Nature. 408, 839841 (2000).

76. J. Lacava, P. Born, T. Kraus, Nanoparticle Clusters with Lennard-Jones Geometries. Nano Lett. 12, 3279-3282 (2012).

77. B. de Nijs et al., Entropy-driven formation of large icosahedral colloidal clusters by spherical confinement. Nat. Mater. 14, 56-60 (2015).

78. G. Brambilla et al., Probing the Equilibrium Dynamics of Colloidal Hard Spheres above the Mode-Coupling Glass Transition. Phys. Rev. Lett. 102, 085703 (2009). 
79. G. L. Hunter, E. R. Weeks, The physics of the colloidal glass transition. Rep. Prog. Phys. 75, 066501 (2012).

80. U. Gasser, A. Schofield, D. A. Weitz, Local order in a supercooled colloidal fluid observed by confocal microscopy. J. Phys. Condens. Matter. 15, S375 (2003).

81. C. P. Royall, A. Malins, A. J. Dunleavy, R. Pinney, Strong geometric frustration in model glassformers. J. Non-Cryst. Solids. 407, 34-43 (2015).

82. S., 1677-1761. Hales, Vegetable staticks: or, an account of some statical experiments on the sap in vegetables: being an essay towards a natural history of vegetation. Also, a specimen of an attempt to analyse the air, By a great Variety of Chymio-Statical Experiments; Which were read at several Meetings before the Royal Society. By Steph. Hales, B. D. F. R. S. Rector of Farringdon, Hampshire, and Minister of Teddington, Middlesex. (printed for W. and J. Innys, at the West End of St. Paul's; and T. Woodward, over-against St. Dunstan's Church in Fleetstreet, London, Electronic reproduction. Farmington Hills, Mich. : Cengage Gale, 2009. Reproduction of original from British Library. Available via World Wide Web., 1727).

83. J. L. Finney, Fine structure in randomly packed, dense clusters of hard spheres. Mater. Sci. Eng. 23, 199-205 (1976).

84. N. Francois, M. Saadatfar, R. Cruikshank, A. Sheppard, Geometrical Frustration in Amorphous and Partially Crystallized Packings of Spheres. Phys. Rev. Lett. 111, 148001 (2013).

85. J. A. van Meel, B. Charbonneau, A. Fortini, P. Charbonneau, Hard-sphere crystallization gets rarer with increasing dimension. Phys. Rev. E. 80, 061110 (2009).

86. A. Malins, J. Eggers, H. Tanaka, C. P. Royall, Lifetimes and lengthscales of structural motifs in a model glassformer. Faraday Discuss. 167, 405-423 (2014).

87. C. P. Royall, S. R. Williams, T. Ohtsuka, H. Tanaka, Direct observation of a local structural mechanism for dynamic arrest. Nat. Mater. 7, 556-561 (2008).

88. A. Malins, S. R. Williams, J. Eggers, H. Tanaka, C. P. Royall, Geometric frustration in small colloidal clusters. J. Phys. Condens. Matter. 21, 425103 (2009).

89. G. Meng, N. Arkus, M. P. Brenner, V. N. Manoharan, The Free-Energy Landscape of Clusters of Attractive Hard Spheres. Science. 327, 560-563 (2010).

90. N. Arkus, V. N. Manoharan, M. P. Brenner, Minimal Energy Clusters of Hard Spheres with Short Range Attractions. Phys. Rev. Lett. 103, 118303-4 (2009).

91. R. S. Hoy, J. Harwayne-Gidansky, C. S. O'Hern, Structure of finite sphere packings via exact enumeration: Implications for colloidal crystal nucleation. Phys. Rev. E. 85, 051403 (2012). 
92. B. Charbonneau, P. Charbonneau, G. Tarjus, Geometrical frustration and static correlations in hard-sphere glass formers. J. Chem. Phys. 138, 12A515 (2013).

93. K. V. Edmond, M. T. Elsesser, G. L. Hunter, D. J. Pine, E. R. Weeks, Decoupling of rotational and translational diffusion in supercooled colloidal fluids. Proc. Natl. Acad. Sci. 109, 17891-17896 (2012).

94. H. S. M. Coxeter, Close-packing and froth. Ill. J. Math. 2, 746-758 (1958).

95. M. Kléman, J. F. Sadoc, A tentative description of the crystallography of amorphous solids. J. Phys. Lett. 40, 569-574 (1979).

96. D. R. Nelson, F. Spaepen, in Solid State Physics, H. Ehrenreich, D. Turnbull, Eds. (Academic Press, 1989; http://www.sciencedirect.com/science/article/pii/S008119470860079X), vol. 42, pp. 1-90.

97. A. Haji-Akbari et al., Disordered, quasicrystalline and crystalline phases of densely packed tetrahedra. Nature. 462, 773-777 (2009).

98. A. Chakrabarty et al., Brownian Motion of Boomerang Colloidal Particles. Phys. Rev. Lett. 111, 160603 (2013).

99. T. Turiv et al., Effect of Collective Molecular Reorientations on Brownian Motion of Colloids in Nematic Liquid Crystal. Science. 342, 1351-1354 (2013).

100. M. C. Marchetti et al., Hydrodynamics of soft active matter. Rev. Mod. Phys. 85, 11431189 (2013).

101. L. Di Michele, E. Eiser, Developments in understanding and controlling self assembly of DNA-functionalized colloids. Phys. Chem. Chem. Phys. 15, 3115-3129 (2013).

102. K.-T. Wu et al., Polygamous particles. Proc. Natl. Acad. Sci. 109, 18731-18736 (2012).

103. J. D. Forster et al., Biomimetic Isotropic Nanostructures for Structural Coloration. Adv. Mater. 22, 2939-2944 (2010).

104. M. Florescu, S. Torquato, P. J. Steinhardt, Designer disordered materials with large, complete photonic band gaps. Proc. Natl. Acad. Sci. 106, 20658 -20663 (2009).

105. T. L. Einstein, T. J. Stasevich, Epitaxial Growth Writ Large. Science. 327, 423-424 (2010).

Acknowledgments: I thank Paul Chaikin, David Grier, David Pine, Mathieu Wyart, Sharon Glotzer, Miranda Holmes-Cerfon, John Crocker, and Paddy Royall for helpful discussions. Research discussed from my own group is funded by the National Science Foundation through grant numbers DMR-1306410 and DMR-1420570. 